\title{
Under Construction - Interactive Project Presentation of a Horticultural Exhibition
}

\author{
Günter Pomaska \\ Faculty for Architecture and Civil Engineering \\ FH Bielefeld, University of Applied Sciences \\ Bielefeld, Germany \\ gp@imagefact.de
}

\begin{abstract}
For information and documentation purposes a multimedia presentation of a horticultural show is installed in a "container" on the construction site of a horticultural show. The "container" is used as a viewing platform and information centre for visitors. For the time being the presentation shows virtual models. Buildings and plantings are presented, as they will appear in future. With the ongoing construction process visual reality, imaged based methods, are used to document the construction work.
\end{abstract}

The interactive presentation can be viewed by operating touch screen monitors or as a large format projection using a data projector. User access is designed in three levels. At the lowest level a slide show is running. Interaction starts by selecting slides from a main menu up to navigation through the local Web page. Access to the operating system and other utilities installed on the Windows platform is protected by software.

Virtual 3d-models are displayed as photo realistic renderings, animations and stereoscopic images. The visitor can follow a low altitude image flight. Aerial photos are shown in high resolution and as orthophoto mosaics. Further information is provided by panoramas, digital images, text and sound.

\section{Introduction}

Oelde, a small town with a population of 30.000 people living in an area of 102 square kilometre in the "Münsterland", a rural district between the cities of Münster and Bielefeld in North-RhineWestphalia, Germany will organize 2001 the "State Horticultural Show". The show is located in an area of 63 ha, divided in the Southern main area which is fenced and a Northern area. The construction includes amongst others the extension of an existing park and water surfaces, restoration of a brook and building of playgrounds for children. Terrain movement of 90.000 cubic metres was necessary.

The multimedia presentation for this project is designed with an interactive user interface in three levels. Starting with an automatically running slide show up to an access through a Web browser. Still keeping in mind that a novice user should operate the presentation on touch screen monitors. A menu structure is designed to divide the content in five chapters: Design, planning and construction site on a time axis, buildings, thematic gardens, variety of plants and events calendar. Articles from newspapers, news and help function are added. A wide range of media presentation techniques like computer graphics, simulation and animation, interactive panoramas, stereoscopic images, text and sound is implemented on PC workstations equipped with touch screen monitors and a data projector for enlarged presentation. 


\section{The "Green Box" and its Hardware Installation}

A small information centre, here called "Green Box", enables access to a viewing platform and an information room. The Green Box is equipped with three computer workstations. Two of them can be operated via touch screen monitors; the other one displays the data via a projector on a large canvas, relatively large in comparison with the monitors. The information area is $12 \times 5$ metre; elevation of the viewing platform is 6.2 metres over ground.

The general configuration of a multimedia terminal is a rugged steel construction and casing with a 15 " TFT-LCD monitor, resolution is $1024 \times 768$ pixel with high colour. Brightness is $250 \mathrm{~cd} / \mathrm{m}^{2}$ and a contrast ration of 300:1. Two wide band speakers are integrated. The "all-in-one" configuration is equipped with the integrated PC, AMD KIII $400 \mathrm{MHz}$ CPU, 512 kByte Cache, 64 MB SDRAM, audio controller, 2MB video DRAM an 6 GB hard disc.

For frequently updating all computers are connected via a local Windows network using 10/100 Base T-Ethernet cards. Installation set up is possible by the CD-Rom drive of the host computer, which is a Compac PC. Canvas surface of the projection area is approx. $2 \times 1.5$ metre. This surface requires a wide-angle lens zoom projector. The canvas surface is limited due to the construction of the "Green Box".


Figure 1: The "Green Box" (here in the background with people visiting the viewing platform) an information centre showing models, displays and computer visualizations. (left)

Figure 2: Touch screen terminal "Displane" installed in the information room of the "Green Box", $500 \mathrm{~mm} \times 1500 \mathrm{~mm} \times 500 \mathrm{~mm}$. (right) 


\section{Protecting Access to System Software by SiteKiosk}

SiteKiosk is an Internet browser supporting the functions of MS Internet Explorer developed for use with public terminals. SiteKiosk is used for exhibitions, libraries, universities or demonstration terminals for example. With a comfortable configuration tool the set up procedure is very easy to handle. It can be predefined what a user is allowed to do and not to do. Access to the operating system is only possible by using a password. Manipulation by using the operating system is therefore excluded for the average user. System shut down by buggy Internet pages is not possible. Error free permanent operating is possible. Touch screen function are supported. The SiteKiosk software changes the terminal into a secure surf station. No attendance is required.

The system is configured for auto start and automatically loads the home page of the presentation in full screen mode and with touch screen support. A keyboard displayed on the screen can be used and scrolling is possible by two additional buttons. Access to other pages as the selected is not allowed. Shut down of the system needs a password. Start up and shut down of the system can be predefined by time functions.

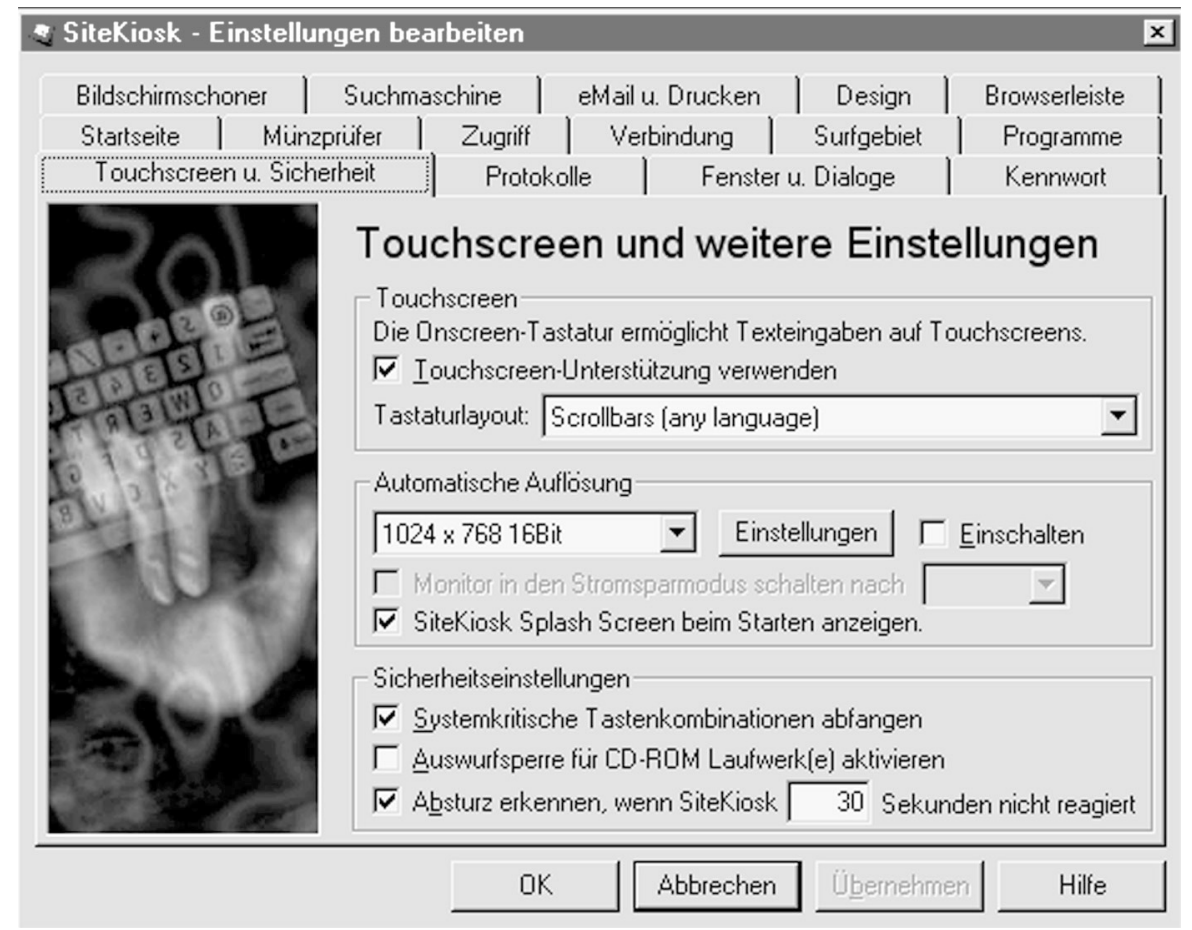

Figure 3: Screenshot of the configuration wizard for SiteKiosk, German version. 


\section{Virtual Reality Models}

Integrated into the landscape design and plantings are a couple of buildings with major influences into the landscape and terrain design. Scene descriptions of these buildings are derived from 2D drawings, sketches and oral descriptions. Since video animations would become very huge data files with limited interaction we decided to produce image sequences and panoramas. Another point of view to avoid video production was the permanent changing of the design and the early state of the presentation. The therefore necessary computing power and time was not justified. Therefore the animation consists of virtual panoramas and simple GIF-animations. Some graphic presentations are played back with background sound recorded on site. Following there are three buildings selected for a short description here.

\subsection{City Tower}

After the design of the "City Tower" realisation was in a long discussion. This building should have been the main attraction of the exhibition. With its height of 25 metres, visiting the tower would give a fine overview about the whole exhibition area. The virtual reality model of the tower was presented by computer graphics and animation but finally the realisation was cancelled. So this is a real virtual model. Figure 4 displays an impression of the first version of the tower. Please note that the original is shown as a stereoscopic image pair to be viewed with red / green glasses or shutter glasses if available.

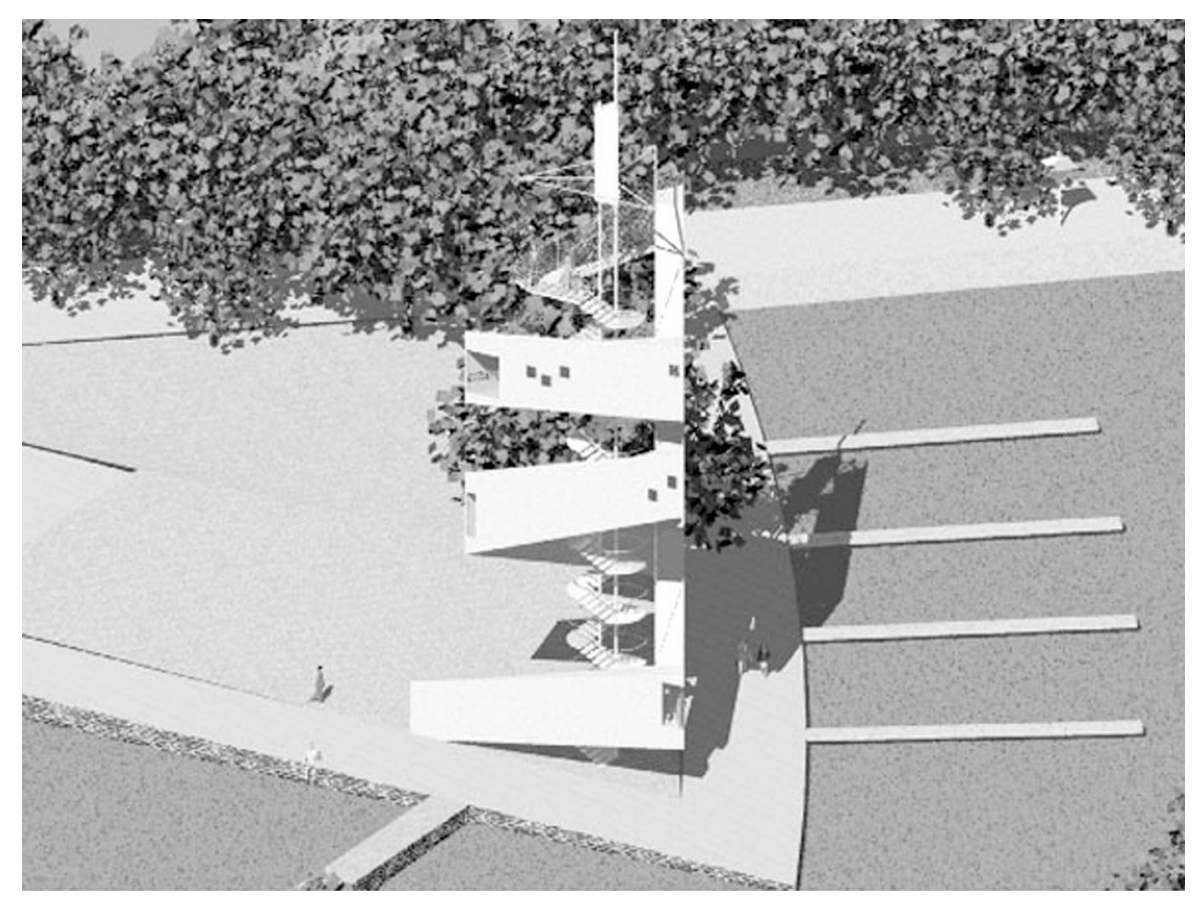

Figure 4: The "City Tower" of the Oiled 2001 Exhibition. A virtual model, not realised.

\subsection{Bird Island}

Another major building is the "bird island". This artificial island is designed with a forest of high bamboo plants, trees called "musische Statisten" and a birdcage. It is difficult to model all the plants tree-by-tree therefore we experimented with surfaces and transparent textures but finally the best impression was realized by reducing the number of trees. Geometric construction of all models was done with AutoCAD and Rhinoceros. Scene descriptions were realized using AccuRender and 3D Studio. 


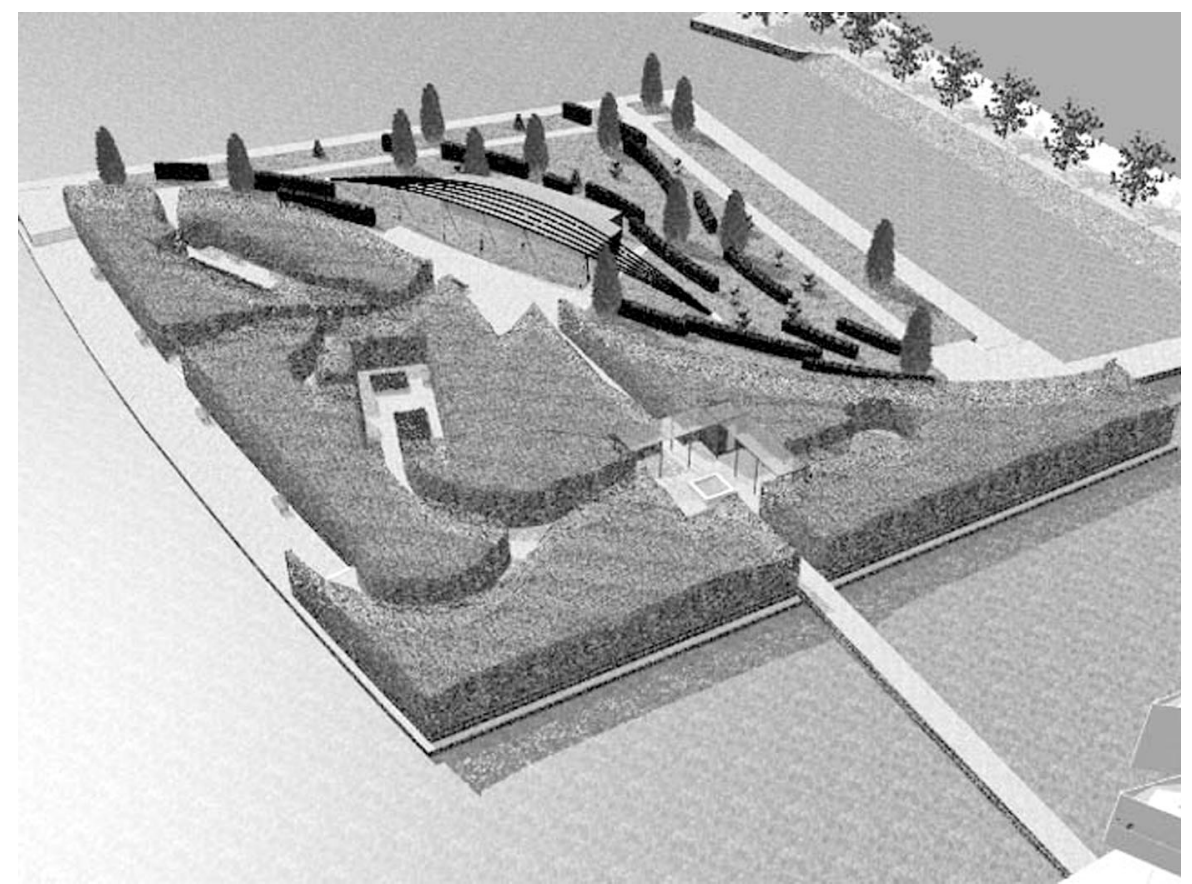

Figure 5: "Bird island" an artificial island with a birdcage and a bamboo forest.

\subsection{Oelder Forum}

The „Oelder Forum“ is projected in the environment of an old water mill. This water mill named "Kramers Mühle" is monument protected by law. Beside this old building the entrance area, a coffee house and a museum for children is designed. The Oelder Forum should become a main communication area of the exhibition. Some scale giving elements like people or street furniture are added to the scene description.

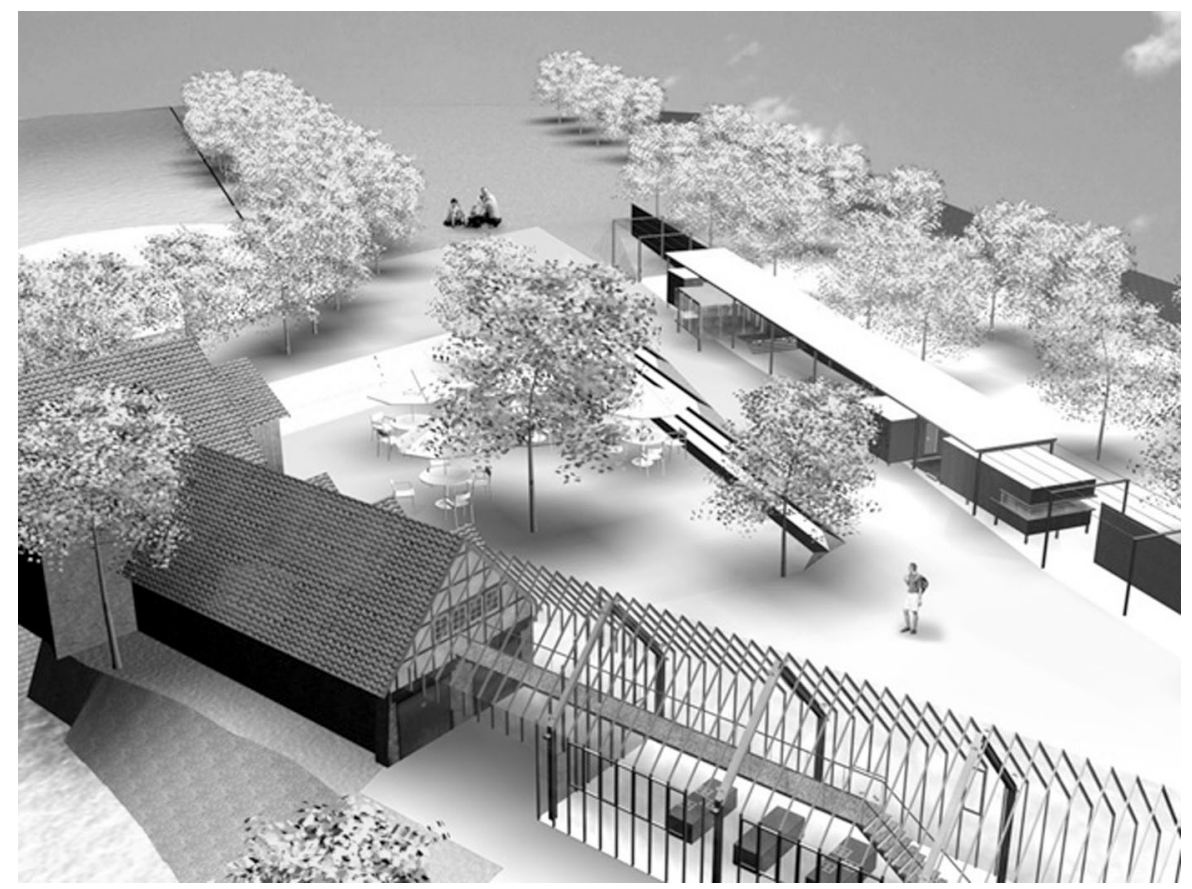

Figure 6: „Oelder Forum“ major communication area of the exhibition, including a monument protected old water mill and a museum for children 


\section{Documentation of the Construction Work Progress}

\subsection{Low Altitude Image Flights}

Planning the horticultural exhibition is based upon large-scale topographic maps, a digital terrain model and orthophotos. An orthophoto is calculated from perspective photos into orthogonal views taking under consideration the geometric shape of the terrain. If the buildings are included in the geometry the orthophoto becomes a true orthophoto. All this material is given by evaluation of a low altitude image flight.

Due to the high degree of automation of digital photogrammetry it is feasible to produce those orthophotos periodically. The orthophotos are displayed by selecting the season from a time axis in the user interface. Figure 6 displays the Southern part of the exhibition. The photo flight was done with LEO (Local Earth Observation) system developed by FH Bochum. The flight height is about $650 \mathrm{~m}$. The equipment used is a Rolleiflex 6006 metric reseau camera with $80 \mathrm{~mm}$ focal length and a bulk-film magazine. The images are then digitised with 2000 ppi and automatically transformed into an orthophoto map with the aid of a Leica Helava System.

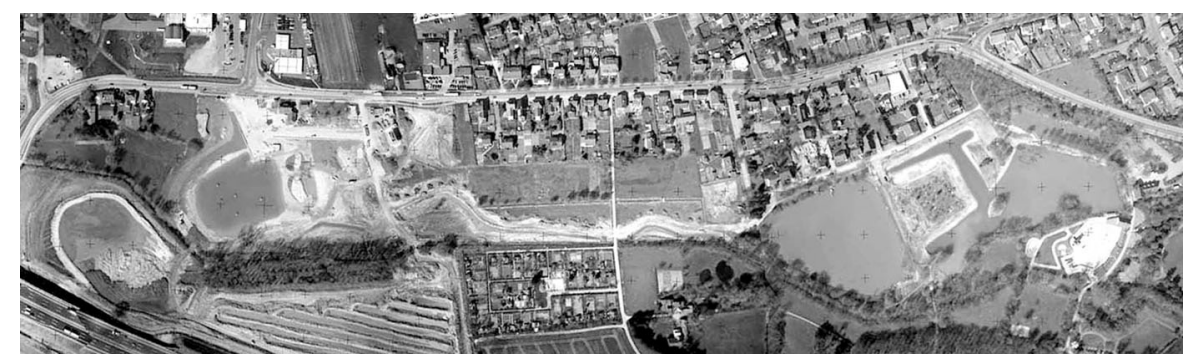

Figure 7: Digital orthophoto of the Southern part of the horticultural exhibition under construction. Pilot and navigator Franz-Josef Heimes.

\subsection{Panoramas}

Interactive viewing of 3D scenes in the Internet is possible with panorama technique, which makes time consuming modelling for the generation of virtual models superfluous. The scene is described entirely on the basis of images. The viewpoint is in the centre of the panorama, the viewing direction can be freely chosen and the viewing distance varied by changing the zoom factor. Segmented panoramas are taken as separate photos from a fixed station with horizontal camera axis and constant rotation. To capture a wider field the camera is usually used in portrait mode. Very precise panoramic views can be obtained with the aid of a special pan head. The digital images are then joined and transformed on an image cylinder or sphere. They are played back in the browser by means of a plug-in, for example MGI ZoomViewer.

To document the work progress panoramas are taken from selected points at specified intervals. The combination of all has been conceived as a Web site, see figure 8. Camera stations are marked in the left-hand menu bar; the time is at the lower edge of the screen, here given in form of tree icons for different seasons. The panorama itself contains the menu bar of the MGI Viewer. 




Figure 8: Screen shot displaying the panorama position menu, a selected panorama and the time axis by tree icons.

\section{Conclusion}

The multimedia presentation described above is not the "big show" and somewhat away from immerse and augmented virtual reality. Facilities on site, time for realisation and as well the budget plays an important role realizing virtual reality. But this presentation and documentation is a first step and documents that computer visualizations are becoming a standard today for information purposes. People wish to know how their environment is influenced by construction. Small-scale models and drawings are for the experts. Interactive virtual reality is for all.

\section{References:}

\section{[1] Pomaska, Günter}

Stereoscopic Images for Visualization of Cultural Heritage

Contribution to ISPRS International Workshop "Photogrammetric Measurement, Object Modelling and Documentation in Architecture and Industry", Thessaloniki, Greece, 1999

\section{[2] Pomaska, Günter}

Documentation and Internet Presentation of Cultural Heritage Using Panoramic Image Technology Contribution to XVII CIPA International Symposium, Recife, Brazil, October 3-6, 1999

[3] Pomaska, Günter

Reconstruction of the Appearance of Schloss Herborn back to 1540 and its Multimedia Presentation ISPRS, Commission V, Amsterdam, 2000

[4] Höhle, Joachim u. Pomaska, Günter

Bygningsmodeller- visualisering og dynamisk praesentation

Arkitekten magasin, marts 2000, 102 argang 
